\title{
Does causal knowledge help us be faster and more frugal in our decisions?
}

\author{
Rocio Garcia-RetaMero \\ Max Planck Institute for Human Development, Berlin, Germany \\ and University of Granada, Granada, Spain \\ ANNIKA Wallin \\ Lund University, Lund, Sweden \\ and Swedish Collegium for Advanced Study in the Social Sciences, Uppsala, Sweden \\ AND \\ AnJa DieckManN \\ Max Planck Institute for Human Development, Berlin, Germany
}

\begin{abstract}
One challenge that has to be addressed by the fast and frugal heuristics program is how people manage to select, from the abundance of cues that exist in the environment, those to rely on when making decisions. We hypothesize that causal knowledge helps people target particular cues and estimate their validities. This hypothesis was tested in three experiments. Results show that when causal information about some cues was available (Experiment 1), participants preferred to search for these cues first and to base their decisions on them. When allowed to learn cue validities in addition to causal information (Experiment 2), participants also became more frugal (i.e., they searched fewer of the available cues), made more accurate decisions, and were more precise in estimating cue validities than was a control group that did not receive causal information. These results can be attributed to the causal relation between the cues and the criterion, rather than to greater saliency of the causal cues (Experiment 3). Overall, our results support the hypothesis that causal knowledge aids in the learning of cue validities and is treated as a meta-cue for identifying highly valid cues.
\end{abstract}

Most of the decisions we make in everyday life have to be fast and are based on minimal information. How else are we to buy groceries or find our soul mate in a crowded bar? How do we make such quick decisions, and how effective are they? The ABC Research Group at the Max Planck Institute for Human Development has suggested that we use fast and frugal heuristics in these situations - that is, simple but, nevertheless, accurate rules for making decisions with a minimum of information (Gigerenzer, Todd, \& the ABC Research Group, 1999; Todd \& Gigerenzer, 2000). These rules are fast because they do not involve much computation, and they are frugal because they search for only some of the available information in the environment.

Some of the fast and frugal heuristics, however, have been criticized for depending on complex computations to select and structure the information they need to be effective. The charge is that these heuristics owe much of their simplicity and success to the computations necessary for setting up the cue search order before they can be used. These computations are very demanding in terms of both memory requirements and computational complexity (Juslin \& Persson, 2002; Wallin \& Gärdenfors, 2000). We suggest that causal knowledge - that is, knowledge about causal relationships between events in the environment- can fill this gap. We explore how this knowledge, in general, might reduce the considerable setup costs of fast and frugal heuristics, and we offer more precise predictions of how it can influence decision-making processes. These predictions were tested in three experiments.

\section{The Fast and Frugal Heuristics Approach: A Critical Review}

One of the fast and frugal heuristics proposed by the $\mathrm{ABC}$ Research Group is take the best (TTB for short), a lexicographic heuristic for two-alternative forced choice tasks (Gigerenzer \& Goldstein, 1996, 1999). ${ }^{1}$ Specifically, TTB is used to infer which of two alternatives, described on several dichotomous cues, has a higher value on a quantitative criterion, such as which of two university professors earns more money, on the basis of such cues as gender or whether the professor teaches at a public or a private university. As a precise step-by-step algorithm, TTB is constructed from building blocks of information gathering and processing to generate a decision. More specifically, this heuristic has a search rule, which prescribes the order in which to search for information (TTB looks up cues in the order of their validity - i.e., the probability that a cue will lead to the correct decision given that it dis-

R. Garcia-Retamero, rretamer@mpib-berlin.mpg.de, rretamer@ugr.es 
criminates between the alternatives; Gigerenzer \& Goldstein, 1996); a stopping rule, describing when the search is to be stopped (TTB stops after the first discriminating cue); and a decision rule for how to use the available cues to make a decision (TTB chooses the alternative favored by the first discriminating cue). Experimental evidence is accumulating suggesting the use of TTB in situations in which information must be searched for in the external environment, especially when there are direct search costs for accessing cues (see Bröder, 2000, 2003; Bröder \& Schiffer, 2003; Garcia-Retamero \& Dieckmann, 2006; Newell \& Shanks, 2003).

These results need to be qualified, however. In most previous experimental studies on the use of TTB, participants were encouraged to use cues in the order of their validity by being informed about cue validities or the validity order (see Bröder, 2000, 2003; Bröder \& Schiffer, 2003; Newell, Weston, \& Shanks, 2003). Without explicit knowledge about cue validities, participants seem to have difficulties computing cue validities and then ordering cues accordingly, even within the confined range of the four to six cues usually available in these experiments (Newell, Rakow, Weston, \& Shanks, 2004; see also Dieckmann \& Todd, 2004; Garcia-Retamero, Takezawa, $\&$ Gigerenzer, 2006). In addition, in life, there are myriad potential cues, and it is impossible to keep track of them all and how they relate to a particular criterion to compute validity (Juslin \& Persson, 2002). Is there a way that the relevant cues can be selected from the abundance of possibilities? If so, how can the validities of those cues be computed? We hypothesize that people do not process all possible cues in their natural environments but, rather, use meta-information, such as causal knowledge, to focus on a small and manageable subset of these cues.

\section{How Causal Knowledge Can Simplify Decision Making}

The adaptive importance of causal processing has been stressed in a wide range of disciplines (see Gopnik \& Schulz, 2007, for a review; see also Garcia-Retamero, Hoffrage, \& Dieckmann, 2007; Garcia-Retamero, Hoffrage, Dieckmann, \& Ramos, 2007). When it is said that a cause brings about an effect, the implication is that there is a stable causal link between the cause and the effect and an underlying causal mechanism that is an essential property of this link (Ahn \& Kalish, 2000; Garcia-Retamero, 2007; GarciaRetamero \& Hoffrage, 2006; Glymour \& Cheng, 1998). Information about causal mechanisms describes what could have mediated (or intervened) between the cause and the effect, thereby enabling the cause to bring about the effect (Ahn, Kalish, Medin, \& Gelman, 1995; Bullock, Gelman, \& Baillargeon, 1982; Koslowski \& Okagaki, 1986). Causal mechanisms go beyond the mere correlation between causes and effects. They distinguish those correlations that are genuinely causal (those in which causal mechanisms do play a role) from those that are spurious (those in which causal mechanisms are absent; Koslowski, 1996; Koslowski, Okagaki, Lorenz, \& Umbach, 1989). In this way, causal knowledge reduces the complexity of representing correlations between events in the environment:
The spurious correlations can be inferred by using information encoded in the causal structure of the environment (Waldmann \& Hagmayer, 2001; Waldmann \& Martignon, 1998).

When it comes to decision making, we posit a twofold benefit for causal knowledge. First, causal knowledge might act as a meta-cue for identifying valid cues in the environment. Second, causal knowledge might help people target particular cues in an otherwise often wide range of possible cues, thus reducing the number of cue-criterion correlations to keep track of when they compute validity by focusing on those cues that are causally linked to the criterion.

As far as the first advantage is concerned, we hypothesize that cues that are causally linked to a criterion tend to be more valid than other cues lacking such a connection to the criterion (see Ahn \& Kalish, 2000; Wallin \& Gärdenfors, 2000). For instance, lung cancer (here, an effect) is more likely to be predicted from a well-established smoking habit (i.e., a cause) than from yellowed fingers (i.e., a second effect of the common cause). Furthermore, correlations between events that are causally linked are likely to be more robust across environments - that is, less sensitive to contextual changes - than are those without such a connection (Pearl, 2000; Reichenbach, 1956). Following our example, the correlation between smoking and lung cancer would be more robust across different patients than the correlation between lung cancer and yellowed fingers would be. We could expect this to be the case even if we control for other alternative causes that could bring about yellowed fingers (e.g., being a painter) that might reduce their predictability for lung cancer. We hypothesize that this asymmetry between causal and noncausal cues that holds in the physical world is reflected in human cognitive processes. We therefore expect decision makers to use their causal knowledge as a meta-cue for selecting highly valid and robust cues in the environment.

Besides facilitating the selection of relevant cues in the environment, causal knowledge might reduce the number of cue-criterion correlations to keep track of when cue validities are computed. This hypothesis is inspired by research using multiple-cue probability-learning tasks. In this paradigm, participants have to predict the criterion of a given object from multiple cues that are probabilistically related to this criterion. Previous empirical studies using this paradigm (see Kruschke \& Johansen, 1999, for a review) suggest that there exist interference effects when multiple cues are available and their validities have to be learned concurrently. For instance, if irrelevant cues are present in such a task, the utilization of valid cues is reduced, and the accuracy of judgments is lower, as compared with a condition in which the irrelevant cues are not included (Castellan, 1973; Edgell \& Hennessey, 1980). One can explain this finding, which can be observed even after a large number of learning trials, by assuming that in the condition with the irrelevant cues, it is harder for participants to identify and focus on the valid cues. In contrast, when participants can learn cue-criterion relationships sequentially - that is, for one cue after anothertheir judgments more closely correspond to the ecological correlations (Brehmer, 1973). On the basis of this finding, 
we suggest that in multiple-cue settings, people equipped with causal knowledge might be able to use this knowledge to focus on certain cues, which, in turn, might facilitate learning of cue validities.

Note, however, that causal knowledge about the cues in the environment also has to be learned. Therefore, our argument holds only if the acquisition of causal knowledge is simpler than the learning of cue validities. There is some evidence for this. First, we know that causal relations are more easily learned and remembered than merely correlational ones (Baumgartner, 1995; Broniarczyk \& Alba, 1994; Koslowski, 1996). Second, we also know that learning of causal Bayes nets, although not necessarily simple, can be simplified if prior specific or abstract domain knowledge about the structure of the environment (e.g., causal directionality) constrains the number of potential causal relations that need to be considered (see Tenenbaum, Griffiths, \& Niyogi, 2007; Waldmann, 1996; Waldmann \& Martignon, 1998). ${ }^{2}$

From these two benefits of causal knowledge, several specific predictions follow regarding the influence of such knowledge on decision-making processes. These predictions focus on search, stopping, and decision rules (i.e., the building blocks of fast and frugal heuristics). Given that causal knowledge might help in identifying highly valid and robust cues in the environment, we hypothesized the following.

1. Search order. Participants would look up cues that were causally connected to the criterion (in short, causal cues) earlier than noncausal cues, even when they had the same validity.

2. Frugality. Participants would stop search earlier and, consequently, look up fewer of the available cues (i.e., they would be more frugal) when causal cues were available than when no causal cues were available.

3. Decision. Participants would rely more on causal cues than on noncausal cues. They would also be more confident and faster in their decisions when causal cues were available than when no causal cues were available.

Because we controlled cue validity in our experiments and thus avoided confounding the existence of causal relationships between cues and the criterion, on the one hand, and cue validity, on the other, causal knowledge could not positively affect participants' accuracy in their decisions. However, given that causal knowledge can reduce the number of cue-criterion relationships to keep track of to compute validity, we hypothesized the following.

4. Estimation of validity. When causal cues were available, participants would be more exact in their validity estimates for causal than for noncausal cues. In addition, if this were the case and participants also showed a preference for causal cues in their decision-making processes, as specified by Hypotheses 1-3, they should also be slightly more accurate in their inferences (since they would be better at identifying causal high-validity cues).

We conducted three experiments to test these hypotheses. The first tested the basic assumption that causal cues are preferred over noncausal cues. The second represented a stronger test by allowing participants to learn the validities of cues in addition to the causal information. The third was devised to rule out the possibility that the results were due to greater saliency of the causal cues (e.g., saliency for linguistic reasons, such as familiarity with the expressions used for describing the cues or their emotional impact), rather than to the causal link between causal cues and the criterion. ${ }^{3}$

\section{EXPERIMENT 1}

\section{Method}

Participants. The participants were 32 undergraduate students at the Free University of Berlin. Nine were male, and 23 were female. They had a mean age of 24 years (range, 19-33). They received $€ 5$ for their participation, plus $50 \%$ of what they earned during the experiment (on average, $€ 10.83$ in total). They were informed about this payoff scheme in the experimental instructions.

Stimuli and Procedure. After reading the instructions, the participants went through a series of computer-based, two-alternative forced choice tasks (five blocks of 20 trials). In each trial, the participants learned about two species of insects and had to decide which would do more damage to a crop. To make this decision, they could inspect up to four cues (i.e., four properties describing the insects: whether or not they had a particular metabolic factor, a long larval phase, a pointed stinger, and conspicuous jaws). These cues were presented by little boxes on the screen that could be clicked to retrieve information. For each cue looked up, 1 cent was deducted from the participants' payoffs. At least one cue had to be looked up to make a decision. The current balance of their account was always visible on the computer screen. When a cue was bought, the cue values of both insects appeared. After each decision, the participants received feedback about whether the decision was correct or not. For a correct decision, they earned 7 cents (minus what they spent on looking up cues). Two of these cues had, counterbalanced across participants, a high validity (i.e., .85), and the other two had a low validity (i.e., .65). All four cues had a discrimination rate 4 of .56. The presence of each cue was independent of that of the other cues (i.e., cue intercorrelation was almost zero).

The experiment was run on two independent groups. In the causal group, the participants were provided with information that causally related two of the cues (also counterbalanced) to the criterion via the experimental instructions (e.g., "the metabolic factor makes the insects hungry and aggressive"). These cues were described in a way that suggested an underlying causal mechanism connecting the cue and the criterion. The two remaining cues were neutral; that is, the way the cues were described did not suggest that the cues were causally related to the criterion (e.g., "the metabolic factor leads to a green and blue coloration of the insect's body"; see Appendix A). Therefore, the participants in the causal group could inspect a causal high-validity cue, a causal low-validity cue, a neutral high-validity cue, or a neutral low-validity cue. In the control group, information about all four cues was neutral. Therefore, two of the cues that the participants could inspect in this group were high-validity cues; the other two were low-validity cues.

Note that the instructions rendering some cues causal (what we will refer to as the causal information) did not in any way explicitly identify these cues as being correlated or causally connected to the criterion, nor did they emphasize them as more important. A pretest, however, confirmed that causal cues-but not neutral cues-were indeed perceived as causally linked to the criterion (see Appendix A). The causal information made it possible for the participants to imagine things that could mediate or intervene between the cues and the criterion but did not enable them to identify which cues were predictive for the criterion. This had to be inferred during the decision phase of the experiment.

In each trial, we recorded six dependent variables: (1) how many cues were looked up in total, (2) which cues were looked up, (3) in which order the cues were looked up, (4) whether the decision was correct or incorrect, (5) how long it took to make a decision (i.e., time between the last cue that was looked up and the decision), and (6) how confident the participants were of their decisions on a scale from 1 (not confident at all) to 6 (very confident). 
Table 1

Percentage of Trials on Which a Causal High-Validity Cue, a Causal Low-Validity Cue, a Neutral High-Validity Cue, and a Neutral Low-Validity Cue Were Looked Up in the First Position in Both the Causal and the Control Groups in Experiments 1, 2, and 3

\begin{tabular}{llcrr}
\hline Group & \multicolumn{1}{c}{ Cues } & Exp. 1 & Exp. 2 & Exp. 3 \\
\hline Causal & Causal high-validity & 43.3 & 52.7 & 56.8 \\
& Causal low-validity & 25.1 & 19.2 & 16.9 \\
& Neutral high-validity & 16.0 & 9.8 & 18.5 \\
& Neutral low-validity & 15.6 & 18.2 & 7.8 \\
Control & Neutral high-validity & 18.4 & 29.8 & 24.0 \\
& Neutral low-validity & 22.8 & 19.1 & 25.3 \\
& Neutral high-validity & 30.9 & 36.7 & 31.7 \\
& Neutral low-validity & 27.9 & 14.5 & 19.0 \\
\hline
\end{tabular}

In addition, after the five blocks of 20 trials of the decision phase, the participants estimated the cue validities. Specifically, for each cue, the participants had to select between two alternatives (i.e., two insects) the one that they thought would show a higher criterion value (i.e., the one that would do more damage to a crop). In one of these alternatives, the cue was present (e.g., it had the metabolic factor). In the other, the cue was absent (e.g., it did not have the metabolic factor). The aim of this question was to control whether or not the participants had learned the cue directions and knew that the presence of a cue indicated a higher criterion value than did its absence. We also asked the participants to tell us in how many out of 100 choices the specific cue (e.g., the insect with the metabolic factor) actually would be associated with a higher criterion value. With this question, we tried to measure to what extent the participants had learned the cues' validities.

\section{Results}

We focused our analyses on the decision-making processes (i.e., search, stopping, and decision processes) and on the participants' estimations of cue validities. In this and the following experiments, we conducted 2 (causal information: causal vs. neutral cues) $\times 2$ (cue validity: high- vs. low-validity cues) $\times 2$ (group: causal vs. control group) ANOVAs to study search and decision processes and estimations of cue validities. For the sake of simplicity and brevity, we will report the effect of the block of trials only when there was a change over time. Consequently, we conducted 3 (block of trials) $\times 2$ (group: causal vs. control group) ANOVAs to analyze frugality and decision accuracy. We used an alpha level of .05 in all the analyses and Tukey's HSD (honest significant difference) test in post hoc analyses.

Search order. The analyses of the percentage of cues that the participants searched in the first position showed a significant interaction between group and causal information $[F(1,30)=5.02, p=.033]$. In accordance with Hypothesis 1 , the participants in the causal group looked up a causal cue first more frequently than they did a neutral cue, regardless of its validity $(p=.044)$. In the control group, the participants did not show a preference for a particular cue in the first position (see Table 1).

Frugality. In disagreement with Hypothesis 2, the groups did not differ in the total number of cues that the participants looked up [2.20 vs. 2.15 cues looked up, on average, in the causal and control groups, respectively; $F(1,30)=0.88, p=.356]$.
Decisions. The analyses of how accurate the participants' responses were showed a main effect of block of trials $[F(4,120)=10.43, p=.001]$. The participants in both the causal and the control groups showed an increase in accuracy across blocks of trials, with $62.5 \%, 69.5 \%, 72.5 \%$, $72.5 \%$, and $74.5 \%$ correct decisions on average for the first to the fifth block, respectively. As a result, an increase in the participants' payoffs was observed across blocks [45 vs. 63 cents in the first and fifth blocks of trials, respectively; $F(4,120)=9.03, p=.001]$. The causal and the control groups did not differ in their average accuracy and payoff.

We further analyzed the proportion of trials on which the participants decided in favor of a certain cue out of all cases in which that cue was looked up and was found to discriminate between options. We also analyzed how confident the participants were of these decisions and how fast they were. Note that these analyses ignore how many cues had been looked up and in what order. Consistent with Hypothesis 3, the analyses showed a significant interaction between group and causal information $[F(1,30)=3.96, p=.048]$. The participants in the causal group decided more often in favor of a causal cue than for a neutral cue (see Table 2). They were also faster in making these decisions, as compared with decisions in favor of a neutral cue [6.59 vs. $7.69 \mathrm{sec} ; F(1,30)=15.60, p<$ $.001]$. Furthermore, the participants were more confident of these decisions, as compared with decisions in favor of a neutral cue $[5.1$ vs. $3.6 ; F(1,30)=8.72, p=.018]$. We need to add that the participants in both the causal and the control groups also decided in favor of a high-validity cue more often than in favor of a low-validity cue $[F(1,30)=$ $3.57, p=.048]$. For confidence and speed of decision making, we found no validity effect.

Estimation of validity. The analyses of the participants' estimations of cue validities showed a main effect of cue validity $[F(1,30)=16.7, p<.001]$, whereby highvalidity cues were judged to be more valid than low-validity cues (see Table 3 ). The interaction between group and causal information was also significant $[F(1,30)=6.02$, $p=.020]$. Only the participants in the causal group evaluated the causal cues as more valid than the neutral cues $(p=.003)$.

To test whether the participants in the causal group were more precise in estimating the validity of the causal cues

Table 2

Percentage of Trials on Which Participants Decided in Accordance With a Causal High-Validity Cue, a Causal Low-Validity Cue, a Neutral High-Validity Cue, and a Neutral Low-Validity Cue in Both the Causal and the Control Groups in Experiments 1, 2, and 3

\begin{tabular}{clccc}
\hline Group & \multicolumn{1}{c}{ Cue } & Exp. 1 & Exp. 2 & Exp. 3 \\
\hline Causal & Causal high-validity & 74.6 & 74.6 & 72.6 \\
& Causal low-validity & 69.0 & 57.7 & 53.8 \\
& Neutral high-validity & 57.7 & 69.0 & 68.7 \\
& Neutral low-validity & 35.9 & 35.9 & 35.3 \\
\multirow{6}{*}{ Control } & Neutral high-validity & 62.1 & 75.1 & 76.4 \\
& Neutral low-validity & 59.2 & 63.0 & 54.7 \\
& Neutral high-validity & 63.0 & 84.1 & 81.6 \\
& Neutral low-validity & 47.2 & 47.2 & 56.9 \\
\hline
\end{tabular}


than that of the neutral cues, we analyzed the absolute difference between estimated and real cue validities. There was a significant interaction between group and causal information $[F(1,30)=4.1, p=.048]$. In line with Hypothesis 4 , the results showed that the participants in the causal group were more precise in estimating the validity of the causal cues than that of the neutral cues ( $p=.048$; see Table 3). The participants' estimations in the control group did not differ depending on the cue. However, this finding needs to be qualified, since the effect might be due to differences in sample size: As was reported above, causal cues were looked up more often than neutral cues, and therefore, their validity estimations can be expected to be more exact, simply for statistical reasons.

\section{Discussion}

In Experiment 1, causal information about cues had an impact on search processes, as well as on decision outcomes, speed, and confidence in decision making. In contrast to the prediction of Hypothesis 2, the participants provided with causal information about some of the cues were not more frugal than those without that information. It could be that these participants explored the environment to test whether causal cues were indeed more valid than those without such a link to the criterion. Since one of the causal cues had a low validity, they had a good reason for reevaluation. This exploratory behavior could have made the groups similar in their frugality. Alternatively, perhaps the participants in the causal group wanted to see all the causal cues, whereas the participants in the control group adopted a different way to limit their information search, restricting themselves to looking up only half of the cues. Both approaches would lead to the same number of cues being looked up (i.e., two). If the lack of frugality differences can be attributed to the causal group's exploratory behavior, as the first explanation suggests, differences should emerge once participants have a chance to learn about cue validities prior to the decision phase. Such a learning phase was implemented in Experiment 2.

\section{EXPERIMENT 2}

In Experiment 2, we went one step further and gave the participants the opportunity to learn the validities of cues in an extensive learning phase before the decision phase, in addition to providing causal information about some of the cues. Therefore, the previous hypotheses should be rephrased in the following way.

Given that causal knowledge might act as a metacue for identifying valuable cues in the environment, we hypothesized the following.

1. Search order. We again expected that the participants would look up cues that were causally connected to the criterion earlier than they would noncausal cues. However, validity would also influence search, because participants would be able to differentiate the causal high-validity cue from the causal low-validity cue throughout the learning phase before the decision phase. Therefore, they would prefer the causal high-validity cue over the rest of the cues.
Table 3

Participants' Validity Estimations for the Causal High-Validity Cue, Causal Low-Validity Cue, Neutral High-Validity Cue, and Neutral Low-Validity Cue (With the Distance Between the Actual and the Estimated Cue Validities in Parentheses) for All Experimental Groups in Experiments 1, 2, and 3

\begin{tabular}{lllll}
\hline Group & \multicolumn{1}{c}{ Cues } & Exp. 1 & Exp. 2 & Exp. 3 \\
\hline Causal & Causal high-validity & $80(10)$ & $82(11)$ & $82(9)$ \\
& Causal low-validity & $73(14)$ & $69(13)$ & $57(12)$ \\
& Neutral high-validity & $65(23)$ & $73(17)$ & $70(18)$ \\
& Neutral low-validity & $54(21)$ & $57(17)$ & $54(19)$ \\
Control & Neutral high-validity & $70(17)$ & $71(15)$ & $68(20)$ \\
& Neutral low-validity & $54(16)$ & $50(19)$ & $57(22)$ \\
& Neutral high-validity & $74(17)$ & $64(21)$ & $67(21)$ \\
& Neutral low-validity & $50(20)$ & $50(21)$ & $55(21)$ \\
\hline
\end{tabular}

2. Frugality. Since the participants would have the opportunity to learn cue validities before the decision phase of the experiment, they would not have to explore the decision environment to test their intuitions about the presumed high validity of the causal cues. Since causal knowledge is expected to foster validity learning in the learning phase through targeting particular cues, we predicted that the participants provided with causal information about some of the cues would be better able to tell the high- and the low-validity causal cues apart, would be more willing to rely on just one cue if it was the high-validity causal cue that discriminated, and thus would be more frugal than the control group participants.

3. Decision. We again expected that the participants would decide in favor of causal cues. However, due to the extensive experience gained during the learning phase, the participants would mostly rely on the causal cue that was highly valid in their decisions.

Given that causal knowledge can reduce the number of cue-criterion relationships to keep track of to compute validity, we expected the following.

4. Estimation of validity. We again hypothesized that the participants would be more accurate in their validity estimates for causal cues than for noncausal ones. We further hypothesized that due to these more accurate estimations of cue validities and a preference for the causal and highly valid cue, the participants in the causal group would now also be more accurate in their decisions.

\section{Method}

Participants. Thirty-two undergraduate students from the Free University of Berlin participated in this experiment. Seventeen were male, and 15 were female. They had a mean age of 24 years (range, 20-37). They received a show-up fee of $€ 8$ for their participation, plus $50 \%$ of what they earned during the experiment (on average, $€ 10.21$ in total). ${ }^{5}$ They were informed about this payoff scheme in the experimental instructions.

Stimuli and Procedure. After reading the instructions informing them about causal/neutral cues (as in the first experiment), the participants went through a learning phase (five blocks of 20 trials) before the decision phase (three blocks of 20 trials). In the learning phase, the values of the four cues were automatically provided at no cost. The participants had to decide which of two alternatives would have a higher criterion value and then received feedback, but they were not paid for their correct decisions in this phase. Other than consisting of just three blocks, the decision phase in Experiment 2 was identical to the deci- 
sion phase in Experiment 1. For the sake of brevity and simplicity and to make these results comparable to those presented in Experiment 1, data analyses will be reported only for the decision phase.

\section{Results}

Search order. The order in which different cues were looked up differed between groups. The analyses of the percentage of cues that the participants searched in the first position showed a significant interaction between group, causal information, and cue validity $[F(1,30)=3.37, p=$ .043]. In the causal group, a causal high-validity cue was more often looked up first than a causal low-validity cue, a neutral high-validity cue, and a neutral low-validity cue $(p \mathrm{~s}=.046, .021$, and .049 , respectively; see Table 1$)$. In the control group, a high-validity cue was more often looked up first than was a low-validity cue $(p=.019)$.

Frugality. The analyses of the total number of cues that the participants searched showed a significant interaction between group and block of trials $[F(2,60)=2.91$, $p=.048]$. The groups did not differ in the total number of cues the participants looked up (2.11 vs. 2.36 for the causal and control groups, respectively). When the block of trials was taken into account, however, the total number of cues that the participants in the causal group looked up decreased, whereas it remained stable in the control group. Consequently, the participants in the causal group were more frugal in the third block than were the participants in the control group (with 2.04 vs. 2.38 cues looked up on average, $p=.049$ ). Therefore, even if the participants did not have to explore the environment to test whether causal cues were indeed more valid than those without such a link to the criterion, they were still reluctant to rely only on the causal high-validity cue and looked up more cues in the first blocks of trials. However, these participants became more frugal over the trials of the decision-making phase.

Decisions. The analyses of how accurate the participants' responses were showed a main effect of block of trials $[F(4,120)=28.46, p<.001]$. The participants in both groups increased their accuracy across trials from $82.5 \%$ to $85.5 \%$ correct decisions $(p=.022)$. The participants in the causal group also tended to make more correct decisions than did the participants in the control group in the final block of trials ( $88 \%$ vs. $82.5 \%, p=$ $.083)$. This result, taken along with the increase in frugality in the causal group, explains why the participants in the causal group obtained a higher overall payoff than did those in the control group [236 vs. 206 cents for the causal and the control groups, respectively; $F(1,30)=$ 4.67, $p=.038]$.

The analyses of the proportion of trials in which the participants decided in favor of a certain cue revealed a significant effect of cue validity $[F(1,30)=25.61, p<$ $.001]$. The participants in both the causal and the control groups decided in favor of a high-validity cue more often than for a low-validity cue (see Table 2 ). The participants in the causal group also decided slightly more often in favor of a causal cue than for a neutral cue (72\% vs. $47 \%)$. However, this difference was not significant $(p=.140)$. We did not find any significant differences in the decision speed or confidence judgments either.
Estimation of validity. Finally, the analyses of the participants' estimations of cue validities showed a main effect of group $[F(1,30)=16.9, p<.001]$, whereby the participants in the causal group judged cues to be more valid than did those in the control group. The main effect of cue validity was also significant $[F(1,30)=39.1, p<$ $.001]$. High-validity cues were judged to be more valid than low-validity cues (see Table 3 ). Furthermore, the analyses of the absolute differences between estimated and real cue validities showed only a significant effect of group $[F(1,30)=4.75, p=.037]$. The participants in the causal group were more precise at estimating cue validities than were the participants in the control group, regardless of whether the cue was causal or neutral, or high or low in validity. Thus, the participants in the causal group were now better overall at estimating cue validities, and unlike in the previous experiment, this difference cannot be attributed to differences in sample size. The participants in the causal group also tended to give more precise estimates of the causal cues' validities than of the neutral cues', although the difference was barely significant (.12 vs. .17 for causal and neutral cues, $p=.059$ ).

\section{Discussion}

Causal knowledge about some of the available cues in the environment had an effect on decision-making processes. Specifically, the participants became more frugal and more accurate in their decisions. They were also more precise in their validity estimations. In contrast to the previous experiment, the participants had the opportunity to learn the actual cue validities before making decisions. As a consequence, they did not rely only on their causal knowledge about the cues, but also on the cues' actual predictive power when they searched and made decisions.

\section{EXPERIMENT 3}

An alternative explanation of the results of our previous experiments is that some cue specifications (what properties a specific metabolic factor leads to, for instance) were for some reasons more salient in their causal than in their neutral versions. Reasons for greater saliency could, for example, be the use of more familiar or more emotionally laden words in the cue descriptions. For instance, it might be easier to notice and remember a metabolic factor that makes insects hungry and aggressive than one that affects the insects' coloration. Therefore, the behavior we observed might have involved participants' searching for salient cues first and basing their decisions on those, rather than searching for causal cues first and basing their decisions on those. The possible higher saliency of the causal cues can also explain why the participants more precisely estimated the validity of these cues rather than that of the neutral ones.

To control for cue saliency, Experiment 3 introduced a set of four cues that could be used to predict two different criteria: which of two species of insects was more likely to pollinate flowers and which was more likely to live longer. Cues existed in two versions. Each version was causally related to only one of the two criteria: Versions of cues that were causal for one criterion were neutral for the other 
criterion, and vice versa. This means that unlike in Experiments 1 and 2, cues were not causal or neutral per se; whether cues were neutral or causal depended entirely on the criterion. For consistency, we will refer to a cue as a causal cue when it is causally related to the criterion and as a neutral cue when it is not. For instance, the information that an insect has a proboscis to defend itself is a causal cue for participants who have to predict survival and a neutral cue for participants who have to predict pollination. In contrast, the information that an insect has a proboscis to get nectar is a causal cue if the criterion is pollination and a neutral cue if the criterion is survival. A pretest confirmed that cues were judged as having a stronger causal effect on the criterion to which they were causally related than on the criterion for which they were neutral (see Appendix B for a full description of cues and criteria and for pretest results). Just as before, two of the four cues had, counterbalanced across participants, a high validity (i.e., .85), and the other two had a low validity (i.e., .65).

The experiment was run on four independent groups: two causal and two control groups. In the first causal group, the participants were asked to predict the first criterion (i.e., living longer) and were given information that causally linked two of the cues to that criterion. The information they received about the remaining two cues did not provide a causal link to the criterion. Therefore, these cues were neutral. Which cues were causal and which were neutral was counterbalanced across participants. In addition, one causal and one neutral cue were given a high validity, and one causal and one neutral cue were given a low validity. Which cues had a high and a low validity was also counterbalanced across participants. The second causal group was identical to the first, except that the two causal cues were linked to the second criterion instead (i.e., pollinating flowers). In the control groups - that is, in the third and fourth groups - all the cues were in their neutral version, and the participants predicted either the first criterion or the second criterion. Again, two cues had a high validity and two cues a low validity, counterbalanced across participants.

Since each version of a cue could be both causal and neutral, any differences in how salient these versions were would affect the relevant control group, as well as the causal group. Thus, if differences between cues were due not to causal knowledge but only to saliency, we should not find differences in decision processes between experimental and control groups. On the other hand, if it was the causal link between cue and criterion that differentiated causal from neutral cues, we should observe differences in search order, frugality, decisions, and validity estimates between the causal and the control groups. We expected that this would be the case. Therefore, our hypotheses for Experiment 3 were identical to those for Experiment 2. We did not expect any differences between the two causal groups or the two control groups.

\section{Method}

Participants. Ninety-six undergraduate students from the Free University of Berlin participated in this experiment. Forty-one were male, and 55 were female. They had a mean age of 24 years (range, 20-28). They received a show-up fee of $€ 8$ for their participation, plus $50 \%$ of what they earned during the experiment (on average, $€ 9.1$ in total). They were informed about this payoff scheme in the experimental instructions.

Stimuli and Procedure. The learning and the decision phases in Experiment 3 were identical to those in Experiment 2. In Experiment 3 , we modified only the information about the cues the participants could use to make a decision and the criterion they had to predict. Data analyses will be reported only for the decision phase.

\section{Results}

We did not find any differences between the two causal groups, on the one hand, and the two control groups, on the other. For clearer presentation, we thus collapsed data from the two causal and the two control groups, respectively.

Search order. In line with the results of Experiment 2, the order in which different cues were looked up differed between groups. The analyses of the percentage of cues that the participants searched in the first position showed a significant interaction between group, causal information, and cue validity $[F(1,94)=6.7, p=.011]$. In the causal groups, a causal high-validity cue was more often looked up first than a causal low-validity cue, a neutral high-validity cue, and a neutral low-validity cue $(p<.001$ for all comparisons; see Table 1). We did not find any significant differences in search order in the control groups. Thus, no cue was preferred over the other cues in these groups.

Frugality. The analyses of the total number of cues that the participants looked up showed a significant interaction between group and block of trials $[F(2,188)=$ $6.91, p=.001]$. Specifically, the total number of cues that the participants in the causal groups looked up decreased over the blocks of trials (2.30 vs. 2.07 looked-up cues, on average, in the first and third blocks of trials, respectively; $p<.001$ ), whereas it remained stable in the control groups ( 2.53 vs. 2.60 cues looked up, on average, in the first and third blocks of trials, respectively; $p=.570$ ). Consequently, the participants in the causal groups were more frugal in the third block of trials than were the participants in the control groups $(p<.001)$.

Decisions. The analyses of how accurate the participants' responses were showed a main effect of block of trials $[F(2,188)=113.1, p<.001]$, whereby the participants in all the groups increased their accuracy across trials from $84 \%$ to $92 \%$ correct decisions $(p<.001)$. The interaction between group and block of trials was also significant $[F(2,188)=15.57, p<.001]$. In the last block of trials, the participants in the causal groups were, with $94 \%$ correct decisions, more accurate than those in the control groups, with $88 \%$ correct decisions $(p<.001)$. Analyses of the proportion of trials on which the participants decided in favor of a certain cue revealed a significant effect of cue validity $[F(1,94)=86.72, p<.001]$. The participants in both the causal and the control groups decided more often in favor of a high-validity cue than for a low-validity cue (see Table 2). However, the participants in the causal group did not decide more often in favor of a causal cue than for a neutral cue $(p=.350)$. We did not find any significant differences in decision speed or confidence judgments. 
Estimation of validity. The analyses of the estimations of cue validities showed a main effect of cue validity $[F(1,94)=95.9, p<.001]$. High-validity cues were judged to be more valid than low-validity cues. The interaction between group and cue validity was also significant $[F(1,94)=$ $7.1, p=.009]$. The participants in the causal groups judged high-validity cues to be more valid than the control groups did $(p<.001)$. The participants in the causal and control groups did not differ in their estimations of the low-validity cues (see Table 3). Furthermore, the analyses of the absolute differences between the estimated and the real cue validities revealed a significant effect of group $[F(1,94)=25.64$, $p<.001]$. The participants in the causal groups were more precise at estimating cue validities than were the participants in the control groups. The interaction between group and causal information was also significant $[F(1,94)=8.02$, $p=.005]$. Thus, the participants in the causal groups were more precise in estimating the validities of the causal cues than in estimating those of the neutral cues.

\section{Discussion}

In Experiment 3, each version of a cue was causal when one criterion was predicted and neutral when the other was predicted. The different versions of the cues were, therefore, encountered in both the causal and the control groups. Thus, potential differences in the saliency of the cue versions cannot explain any differences between the causal and the control groups. However, we did find differences between groups: Only the participants in the causal groups preferred to start by looking up the causal high-validity cue. More important, the participants became more frugal over trials and more accurate in their decisions only when cues that had a causal link to the criterion were available. Overall, the results of Experiments 2 and 3 are almost identical, and both sets of results support the thesis that causal knowledge helps people target particular cues and estimate their validities.

\section{GENERAL DISCUSSION}

In three experiments, we examined the impact of providing causal information about cue-criterion relationships on the decision-making processes in two-alternative forced choice tasks. In Experiment 1, one group of participants, the causal group, was provided with instructions that causally related two of the cues that could be used to make a decision to the criterion. In the control group, the participants received neutral information about all four cues. In Experiments 2 and 3, the participants in both the causal group(s) and the control group(s) were allowed to learn cue validities in addition to receiving causal/neutral information about them.

The results of Experiment 1 showed that when causal information about some of the cues was available, the participants preferred to start searching with a causal cue, regardless of its validity. They also decided in favor of causal cues more often than neutral ones and were faster and more confident in their decisions than when they did not have causal cues to rely on. Finally, the participants were more precise in estimating the validities of the causal cues than those of the neutral ones. These results are in accordance with our
Experimental Hypotheses 1, 3, and 4. We interpret them as evidence that the participants perceived causal information as indicative of highly valid cues in the environment, since they gave priority to causal cues in both the search and the decision processes. Furthermore, causal knowledge seems to facilitate cue validity learning, since the participants in the causal group were more precise in their validity estimations for causal than for noncausal cues. However, this result could be due to differences in sample size, because the participants preferred to search causal cues over the rest of the cues. Hypothesis 2 was not supported in Experiment 1. Specifically, the participants in the causal group were not more frugal than those in the control group. They obviously did not rely on causal cues exclusively.

In Experiment 2, we allowed the participants to learn the validity of the cues. Supporting our hypothesis, search processes were influenced by both causal information and validity. Specifically, the participants in the causal group preferred to start by looking up the causal high-validity cue. Furthermore, these participants now became more frugal over trials, as well as more accurate in their decisions. They also achieved a higher precision on average (i.e., across all cues) in estimating cue validities, especially for the causal ones. Overall, the increasing frugality and accuracy in the causal group led to a higher final payoff than for the control group.

In Experiment 3, we analyzed whether our previous results could be attributed to a higher saliency of the causal cues, regardless of any possible causal relation between these cues and the criterion. To test this alternative explanation, we introduced two criteria and used each cue as both a causal and a neutral cue. Thus, potential differences in the saliency between cues would have affected both the causal and the control groups. Yet we found that only the participants in the causal groups preferred to start by looking up the causal high-validity cue. Furthermore, only these participants became more frugal over trials and more accurate in their decisions. We conclude that the causal relation between cue and criterion helps people target particular cues and estimate their validities.

In sum, our experimental results showed that information about the causal relations between cues and criterion was used as a meta-cue for identifying and selecting cues in the environment. It also helped the participants target a manageable subset of cues in the environment and, consequently, led to more accurate learning of cue validities.

The adaptive value of causal knowledge. The human capacity to assess relationships between events has long been a topic of interest in the area of judgment and decision making (for reviews, see Alloy \& Tabachnik, 1984; Murphy, 2002; Shanks, Holyoak, \& Medin, 1996). One interesting effect consistent with our results is that judgments of correlation are strongly influenced by prior knowledge (Alba \& Hasher, 1983). For instance, Fugelsang and Thompson (2003; see also Fugelsang \& Thompson, 2000) showed that people's preexisting beliefs about causal relationships between events help them to restrict and select the set of cues for which covariation is considered. This tendency seems to have beneficial effects. If used as hypotheses to be tested on data, prior expectations can increase the accuracy of 
covariation judgments: Assessments of covariation that are guided by such theories are more accurate than theory-free judgments about abstract stimuli (Baumgartner, 1995; but see Nisbett \& Ross, 1980). Such theories also result in more robust judgments of covariation by reducing the impact of atypical data points (Wright \& Murphy, 1984). A related phenomenon has also been observed in the area of categorization. Specifically, people fail to form categories on the basis of correlated attributes unless a conceptual basis for their correlation can be found (Medin, Wattenmaker, \& Hampson, 1987; see Medin, 1989, for a review).

Our results are also in line with the research on Simpson's paradox 6 in causal learning. For instance, in a series of experiments, Spellman, Price, and Logan (2001; see also Waldmann \& Hagmayer, 2001) considered causal structures involving a potential cause, an effect, and a third related event. In these experiments, participants had to estimate the effectiveness of the potential cause to produce the effect on the basis of the covariation between them. Interestingly, the assumed causal role of the third event crucially determined whether it was taken into account when these causal judgments were made. Specifically, when the participants had no reason to believe that the third event was causally linked to the effect, they reported the effectiveness of the potential cause without taking the presence of the third event into account. However, when they knew that the third event was also causally linked to the effect, they reported the effectiveness of the potential cause conditional on the presence or absence of the third (causal) event. Briefly, the participants' causal knowledge determined which information they paid attention to when making causal estimations (see also Schaller, 1992, and Schaller \& O’Brien, 1992, for similar results in stereotype formation). In sum, research in all these domains suggests that covariation assessment is facilitated by causal knowledge.

Causal knowledge reduces the setup costs of fast and frugal heuristics. Seen through the lens of the fast and frugal heuristic framework, causal knowledge helps people select valid cues to be placed in a high position in the cue ordering - that is, in the hierarchy that is accessed by the search process of a heuristic. If feedback about whether a decision was correct or incorrect is available, the cue ordering might be updated. In this sense, causal beliefs can be perceived as hypotheses to be tested and updated with empirical data (see also Koslowski, 1996; Koslowski \& Masnick, 2002). For instance, some of the selected causal cues might turn out to be highly valid cues. Such cues would remain in a high position in the cue ordering. Consequently, the expected usefulness of the causal beliefs that link those cues to the criterion would be strengthened. However, other causal cues might turn out to have low validity. The expected usefulness of the causal belief that links those cues to the criterion would therefore be reduced, and those cues would end up in a lower position in the cue ordering. In short, causal beliefs might act as hypotheses that constrain the cues that are selected to make decisions, and these beliefs would subsequently be confirmed or disconfirmed on the basis of the experience with the selected cues in the environment.

As was explained above, in most previous experimental studies of the use of the lexicographic heuristic TTB, participants did not have to assess the correlation between cues and criterion on their own but were informed about cue validities or the validity order (e.g., Bröder, 2000, 2003; Bröder \& Schiffer, 2003; Newell et al., 2003). When this was not the case, people, for the most part, did not come up with a cue order by validity (Newell et al., 2004). However, in all these experiments, the influence of participants' causal knowledge about the cues was eliminated by making experimental cues neutral. People therefore could not use their causal knowledge about variables in the environment to identify valid cues and to reduce the number of cues to a more manageable subset for tracing their performance.

A risk with eliminating causal information from an experiment is that decision makers might appear less competent than they really are. Stripping the experimental task of content that is meaningful to participants will distort the strategies they use, since they no longer are representative of the everyday situations for which they are designed. In our set of controlled experiments, we have identified one meaningful factor, causal information, that changes how participants learn cue validities, how they search for information, and how accurate their decisions are. Causal knowledge is a vital part of how people decide which cues to trust.

\section{AUTHOR NOTE}

We thank Barbara Spellman, Michael Oaksford, Brian Ross, Ulrich Hoffrage, Lael Schooler, Magnus Persson, Peter Todd, and an anonymous reviewer for their helpful discussions. We thank Angel Garcia-Retamero for his help in writing the program for the experiments. We also thank Anita Todd and Donna Alexander for editing the manuscript. In addition, the second author gratefully acknowledges the Bank of Sweden Tercentenary Foundation and the Swedish Collegium for Advanced Study in the Social Sciences. Correspondence concerning this article should be addressed to R. Garcia-Retamero, Max Planck Institute for Human Development, Lentzeallee 94, D-14195 Berlin, Germany (e-mail: rretamer@, mpib-berlin.mpg.de, rretamer@ugr.es).

\section{REFERENCES}

Ahn, W., \& Kalish, C. W. (2000). The role of mechanism beliefs in causal reasoning. In F. C. Keil \& R. A. Wilson (Eds.), Explanation and cognition (pp. 199-225). Cambridge, MA: MIT Press.

Ahn, W., Kalish, C. W., Medin, D. L., \& Gelman, S. A. (1995). The role of covariation versus mechanism information in causal attribution. Cognition, 54, 299-352.

Alba, J. W., \& HASHER, L. (1983). Is memory schematic? Psychological Bulletin, 93, 203-231.

Alloy, L. B., \& TABACHNiK, N. (1984). Assessment of covariation by humans and animals: The joint influence of prior expectations and current situational information. Psychological Review, 91, 112-149.

BaumgarTner, H. (1995). On the utility of consumers' theories in judgments of covariation. Journal of Consumer Research, 21, 634-643.

Brehmer, B. (1973). Note on the relation between single-cue probability learning and multiple-cue probability learning. Organizational Behavior \& Human Performance, 9, 246-252.

BRöDER, A. (2000). Assessing the empirical validity of the "take-the-best" heuristic as a model of human probabilistic inference. Journal of Experimental Psychology: Learning, Memory, \& Cognition, 26, 1332-1346.

BRÖDER, A. (2003). Decision making with the "adaptive toolbox": Influence of environmental structure, intelligence, and working memory load. Journal of Experimental Psychology: Learning, Memory, \& Cognition, 29, 611-625.

BRÖDER, A., \& SCHIFFER, S. (2003). Take the best versus simultaneous feature matching: Probabilistic inferences from memory and effects of representation format. Journal of Experimental Psychology: General, 132, 277-293.

BroniarcZyK, S. M., \& Alba, J. W. (1994). Theory versus data in pre- 
diction and correlation tasks. Organizational Behavior \& Human Decision Processes, 57, 117-139.

Bullock, M., Gelman, R., \& Baillargeon, R. (1982). The development of causal reasoning. In W. J. Friedman (Ed.), The developmental psychology of time (pp. 209-254). New York: Academic Press.

Castellan, N. J. (1973). Multiple-cue probability learning with irrelevant cues. Organizational Behavior \& Human Performance, 9, 16-29.

Dieckmann, A., \& Todd, P. M. (2004). Simple ways to construct search orders. In K. Forbus, D. Gentner, \& T. Regier (Eds.), Proceedings of the 26th Annual Conference of the Cognitive Science Society (pp. 309314). Mahwah, NJ: Erlbaum.

Edgell, S. E., \& Hennessey, J. E. (1980). Irrelevant information and utilization of event base rates in nonmetric multiple-cue probability learning. Organizational Behavior \& Human Performance, 26, 1-6.

Eells, E. (1991). Probabilistic causality. Cambridge: Cambridge University Press.

Fugelsang, J. A., \& Thompson, V. A. (2000). Strategy selection in causal reasoning: When beliefs and covariation collide. Canadian Journal of Experimental Psychology, 54, 15-32.

Fugelsang, J. A., \& Thompson, V. A. (2003). A dual-process model of belief and evidence interactions in causal reasoning. Memory \& Cognition, 31, 800-815.

Garcia-Retamero, R. (2007). The influence of knowledge about causal mechanisms on compound processing. Psychological Record, 57, 295-306.

Garcia-Retamero, R., \& Dieckmann, A. (2006). A critical view of the fast and frugal heuristics approach. Revista Latinoamericana de Psicología, 38, 509-522.

Garcia-Retamero, R., \& Hoffrage, U. (2006). How causal knowledge simplifies decision making. Minds \& Machines, 16, 365-380.

Garcia-Retamero, R., Hoffrage, U., \& Dieckmann, A. (2007). When one cue is not enough: Combining fast and frugal heuristics with compound cue processing. Quarterly Journal of Experimental Psychology, 60, 1197-1215.

Garcia-Retamero, R., Hoffrage, U., Dieckmann, A., \& Ramos, M. (2007). Compound cue processing within the fast and frugal heuristic approach in non-linearly separable environments. Learning \& Motivation, 38, 16-34.

Garcia-Retamero, R., Takezawa, M., \& Gigerenzer, G. (2006). How to learn good cue orders: When social learning benefits simple heuristics. In R. Sun \& N. Miyake (Eds.), Proceedings of the 28th Annual Conference of the Cognitive Science Society (pp. 1352-1358). Mahwah, NJ: Erlbaum.

Gigerenzer, G., \& Goldstein, D. G. (1996). Reasoning the fast and frugal way: Models of bounded rationality. Psychological Review, 103, 650-669.

Gigerenzer, G., \& Goldstein, D. G. (1999). Betting on one good reason: The take the best heuristic. In G. Gigerenzer, P. M. Todd, $\&$ the ABC Research Group, Simple heuristics that make us smart (pp. 75-95). New York: Oxford University Press.

Gigerenzer, G., Todd, P. M., \& THe ABC Research Group (1999). Simple heuristics that make us smart. New York: Oxford University Press.

Glymour, C., \& Cheng, P. W. (1998). Causal mechanism and probability: A normative approach. In M. Oaksford \& N. Chater (Eds.), Rational models of cognition (pp. 295-313). Oxford: Oxford University Press.

GopNik, A., \& Schulz, L. (2007). Causal learning: Psychology, philosophy, and computation. Oxford: Oxford University Press.

Juslin, P., \& Persson, M. (2002). Probabilities from exemplars (PROBEX): A "lazy" algorithm for probabilistic inference from generic knowledge. Cognitive Science, 26, 563-607.

Koslowski, B. (1996). Theory and evidence: The development of scientific reasoning. Cambridge, MA: MIT Press.

KosLowsKi, B., \& MASNICK, A. (2002). The development of causal reasoning. In U. Goswami (Ed.), Blackwell handbook of childhood cognitive development (pp. 257-281). Malden, MA: Blackwell.

KoslowsKi, B., \& OKAGAKI, L. (1986). Non-Humean indices of causation in problem-solving situations: Causal mechanism, analogous effects, and the status of rival alternative accounts. Child Development, 576, 1100-1108.

Koslowski, B., Okagaki, L., Lorenz, C., \& Umbach, D. (1989). When covariation is not enough: The role of causal mechanism, sampling method, and sample size in causal reasoning. Child Development, $6 \mathbf{6}$, 1316-1327.
Kruschke, J. K., \& Johansen, M. K. (1999). A model of probabilistic category learning. Journal of Experimental Psychology: Learning, Memory, \& Cognition, 25, 1083-1119.

Medin, D. L. (1989). Concepts and conceptual structure. American Psychologist, 44, 1469-1481.

Medin, D. L., Wattenmaker, W. D., \& Hampson, S. E. (1987). Family resemblance, conceptual cohesiveness, and category construction. Cognitive Psychology, 19, 242-279.

MurPHY, G. L. (2002). The big book of concepts. Cambridge, MA: MIT Press.

Newell, B. R., Rakow, T., Weston, N. J., \& Shanks, D. R. (2004). Search strategies in decision making: The success of "success." Journal of Behavioral Decision Making, 17, 117-137.

Newell, B. R., \& Shanks, D. R. (2003). Take the best or look at the rest? Factors influencing "one-reason" decision making. Journal of Experimental Psychology: Learning, Memory, \& Cognition, 29, 53-65.

Newell, B. R., Weston, N. J., \& Shanks, D. R. (2003). Empirical tests of a fast-and-frugal heuristic: Not everyone "takes-the-best." Organizational Behavior \& Human Decision Processes, 91, 82-96.

NisBetT, R. E., \& Ross, L. (1980). Human inference: Strategies and shortcomings of social judgments. Englewood Cliffs, NJ: Prentice-Hall.

Pearl, J. (2000). Causality. New York: Oxford University Press.

Reichenbach, H. (1956). The direction of time. Berkeley: University of California Press.

Schaller, M. (1992). In-group favoritism and statistical reasoning in social inference: Implications for formation and maintenance of group stereotypes. Journal of Personality \& Social Psychology, 63, 61-74.

SCHALler, M., \& O'Brien, M. (1992). Intuitive analysis of covariance and group stereotype formation. Personality \& Social Psychology Bulletin, 18, 776-785.

Shanks, D. R., Holyoak, K. J., \& Medin, D. L. (1996). The psychology of learning and motivation (Vol. 34). San Diego: Academic Press.

Spellman, B. A., Price, C. M., \& Logan, J. M. (2001). How two causes are different from one: The use of (un)conditional information in Simpson's paradox. Memory \& Cognition, 29, 193-208.

SPIRTES, P., Glymour, C., \& Scheines, R. (1993). Causation, prediction, and search (Springer lecture notes in statistics). New York: Springer.

Spirtes, P., Glymour, C., \& Scheines, R. (2000). Causation, prediction, and search (2nd ed.). Cambridge, MA: MIT Press.

Tenenbaum, J. B., Griffiths, T. L., \& NiYogi, S. (in press). Intuitive theories as grammars for causal inference. In A. Gopnik \& L. Schulz (Eds.), Causal learning: Psychology, philosophy, and computation. Oxford: Oxford University Press.

Todd, P. M., \& Gigerenzer, G. (2000). Précis of simple heuristics that make us smart. Behavioral \& Brain Sciences, 23, 727-780.

WaLdmanN, M. R. (1996). Knowledge-based causal induction. In D. R. Shanks, K. J. Holyoak, \& D. L. Medin (Eds.), The psychology of learning and motivation (Vol. 34, pp. 47-88). San Diego: Academic Press.

WALDMANN, M. R., \& HAGMAYER, Y. (2001). Estimating causal strength: The role of structural knowledge and processing effort. Cognition, 82, 27-58.

Waldmann, M. R., \& Martignon, L. (1998). A Bayesian network model of causal learning. In M. A. Gernsbacher \& S. J. Derry (Eds.), Proceedings of the 20th Annual Conference of the Cognitive Science Society (pp. 1102-1107). Mahwah, NJ: Erlbaum.

WALLIN, A., \& GäRDENFORS, P. (2000). Smart people who make simple heuristics work. Behavioral \& Brain Sciences, 23, 765.

Wright, J. C., \& Murphy, G. L. (1984). The utility of theories in intuitive statistics: The robustness of theory-based judgments. Journal of Experimental Psychology: General, 113, 301-322.

\section{NOTES}

1. We will use TTB as an example of a fast and frugal heuristic throughout the article, but we believe that our reasoning also applies to other fast and frugal heuristics, which have in common that they rely on few cues to make a decision and, therefore, need to be choosy in selecting the right ones.

2. Research in the field of artificial intelligence has recently proposed a number of algorithms capable of easily inferring causal relations from covariation patterns (e.g., the TETRAD II program; Spirtes, Glymour, \& Scheines, 1993, 2000). These algorithms use causal models to generate a certain pattern of statistical dependencies and then search for those clues 
that reveal fragments of the underlying structure. These fragments are pieced together to form a coherent causal model. Obviously, these systems do not provide information about how humans learn causal links, but they do tell us how such a task might be solved.

3. We thank Mike Oaksford and an anonymous reviewer for pointing out this alternative explanation of the results of Experiments 1 and 2.

4. The discrimination rate of a cue is the proportion of pair comparisons in which the value of that cue in the two decision alternatives differs (Gigerenzer \& Goldstein, 1996).
5. The show-up fee in Experiments 2 and 3 was higher than that in Experiment 1 because the participants were expected to invest more time in the task, with less opportunity to earn money because the decision phase was shorter.

6. The Simpson's paradox (Eells, 1991; Pearl, 2000) describes the fact that a given correlation between two events that holds in a given population can disappear or even be reversed in all subpopulations when the population is partitioned in certain ways (cf. Waldmann \& Hagmayer, 2001)

\section{APPENDIX A}

Materials Used in Experiments 1 and 2: Causal and neutral versions of the four properties that participants could use to determine which of two species of insects would do more damage to a crop.
Cue
Causal Version
Neutral Version

Some insects have a metabolic

factor in their blood.

Some insects have a

long larval phase.

Some insects have a pointed stinger.

Some insects have conspicuous jaws.
The metabolic factor makes the insects hungry and aggressive.

The insects have a greatly increased need for nutrition in the larval phase.

The insects use the stinger to lay eggs in plants.

The jaws are used for breaking even hard parts of the plants into pieces.

The metabolic factor leads to
a green and blue coloration of
the insects' body.
The insects have to be
protected by adult conspecifics
in the larval phase.
The insects sometimes use the
stinger to prick humans.
The jaws are used in
competitive fights.
The metabolic factor leads to a green and blue coloration of insects' body.

The insects have to be in the larval phase. stinger to prick humans. competitive fights.

In a pretest, participants $(n=42)$ rated how strongly a cue causally affected the criterion, either in its causal or its neutral version, on a scale from 1 to 5 (with 5 being the strongest effect). Results show that a cue was judged as having a stronger causal effect on the criterion in its causal version than in its neutral version [4.30 vs. 1.56; $F(1,40)=107.96, p<.001]$. This result stands in stark contrast to comparisons between cues within each of the two versions. In their causal versions, there was no difference between cues in how strongly causal cues were perceived to affect the criterion $[F(3,60)=2.11, p=.189]$. The same was true for neutral cues $[F(3,60)=1.48$, $p=.228]$. Consequently, this procedure guarantees that the differences in decision-making processes between the causal and the control experimental groups can be attributed only to the perceived causal relation between the causal cues and the criterion in the causal group.

\section{APPENDIX B}

Materials Used in Experiment 3: Causal and neutral versions of the four properties that participants could use to determine which of two species will live longer. When the criterion shifted to determining which insect was more likely to pollinate flowers, causal cues become neutral and vice versa.

\begin{tabular}{|c|c|c|}
\hline Cue & $\begin{array}{l}\text { Causal Version for "Living Longer" and } \\
\text { Neutral for "Pollinating Flowers" }\end{array}$ & $\begin{array}{l}\text { Neutral Version for "Living Longer" } \\
\text { and Causal for "Pollinating Flowers" }\end{array}$ \\
\hline $\begin{array}{l}\text { Some insects have a } \\
\text { metabolic factor in } \\
\text { their blood. }\end{array}$ & $\begin{array}{l}\text { The metabolic factor makes the insects' } \\
\text { coloration brown, allowing them to } \\
\text { blend into the soil. }\end{array}$ & $\begin{array}{l}\text { The metabolic factor makes the } \\
\text { insects move around a lot. }\end{array}$ \\
\hline $\begin{array}{l}\text { Some insects have } \\
\text { a larval phase. }\end{array}$ & The larvae live in hard shells. & The larvae live in flowers. \\
\hline $\begin{array}{l}\text { Some insects have } \\
\text { a pointed proboscis. }\end{array}$ & $\begin{array}{l}\text { The insects use the proboscis } \\
\text { to defend themselves. }\end{array}$ & $\begin{array}{l}\text { The insects use the proboscis } \\
\text { to get nectar. }\end{array}$ \\
\hline $\begin{array}{l}\text { Some insects have } \\
\text { conspicuous jaws. }\end{array}$ & $\begin{array}{l}\text { The insects use the jaws for hanging on } \\
\text { to plants when there are tropical storms. }\end{array}$ & $\begin{array}{l}\text { The insects use the jaws } \\
\text { to climb plants. }\end{array}$ \\
\hline
\end{tabular}

Pretest results $(n=60)$ show that cues were judged as having a stronger causal effect on the criterion to which they were causally related than on the criterion for which they were neutral [4.26 vs. 2.58 on a scale from 1 to 5 , with 5 being the strongest effect; $F(1,224)=194.9, p<.001]$. In their causal versions, there was no difference between cues in how strongly they were perceived to affect the criterion regardless of which criterion was predicted $[F(3,113)=0.31, p=.819]$. The same was true for the cues in their neutral versions $[F(3,113)=1.59, p=.189]$. 